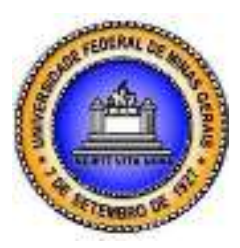

\title{
ANÁLISE DAS REPROVAÇÕES DE MATERIAIS MÉDICO-HOSPITALARES DE UM HOSPITAL DE ENSINO
}

ANALYSIS OF DEPRECATIONS OF A MEDICAL MATERIALS TEACHING HOSPITAL

ANÁLISIS DE DENEGACIONES DE MATERIALES MÉDICOS DE UN HOSPITAL DE ENSEÑANZA

Luiz Binicio dos Reis

Universidade Estadual de Montes Claros

carla_silvana@santoagostinho.edu.br

Isabelle Arruda Barbosa

Universidade Estadual de Montes Claros

isabelle_arruda@yahoo.com.br

Lucas José Ferreira Soares

Universidade Estadual de Montes Claros

carla_silvana@santoagostinho.edu.br

Iara Marina da Cruz

Universidade Estadual de Montes Claros

iara.marina@yahoo.com.br

Luís Paulo Souza e Souza

Universidade Estadual de Montes Claros

$\underline{\text { luis.pauloss@hotmail.com }}$

Carla Silvana de Oliveira e Silva

Universidade Estadual de Montes Claros

carlasosilva@ig.com.br

Dulce Aparecida Barbosa

Universidade Estadual de Montes Claros

carlasosilva@ig.com.br
Submetido em: 04/04/2014

Artigo aceito em: 04/05/2015

\section{RESUMO}

Objetivou-se identificar os motivos de reprovações de materiais médico-hospitalares (MMH) no edital de licitação 001 e 029 /2011, na modalidade pregão eletrônico e identificar as categoriais profissionais envolvidas na avaliação da qualidade dos materiais médico-hospitalares. Trata-se de um estudo quantitativo de caráter descritivo, transversal e documental. Os dados foram obtidos a partir dos formulários utilizados pelos profissionais com intuito de averiguar a qualidade de tais materiais no edital de licitação na modalidade pregão eletrônico 001 e 029 /2011, presentes no arquivo da Comissão de Padronização de Materiais e Produtos (COPAMP) de hospital universitário ao norte de Minas Gerais. Observou-se que os motivos de reprovações dos MMH relacionaram-se à baixa qualidade técnica do produto e o profissional enfermeiro constitui-se num ponto forte nesses dois processos licitatórios. A estratégia de submeter os $\mathrm{MMH}$ em fase de pré-aquisição à avaliação da qualidade junto aos profissionais é uma das ações mais preventivas e eficazes dentro do contexto da administração de materiais no ambiente hospitalar, uma vez que previne aquisição de produtos de baixa qualidade. 

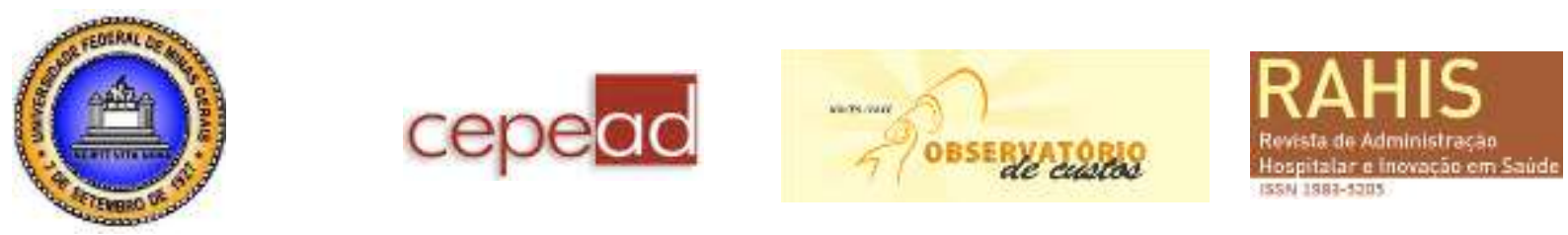

Palavras chave: Inspeção de Qualidade; Compras Hospitalares; Gerenciamento de segurança; Administração de materiais no hospital.

\begin{abstract}
Aimed to identify the reasons of deprecations of medical materials (MM) in the notice with invitation to bid 001 and 029/2011, electronic bidding mode and identify the professional categories involved in evaluating the quality of medical materials. It is a quantitative study of descriptive, transversal and documentary character. The data were obtained from the forms used by professionals in order to ascertain the quality of such materials in the notice with invitation to bid in electronic bidding 001 and 029 2011, present in the file of the Commission for Standardization of materials and products of teaching hospital in North of Minas Gerais. It was observed that the grounds of deprecations of the MM related to low technical quality of the product and the professional nurse constitutes a strong point in those two bidding processes. The strategy of submitting the MM on pre-acquisition phase to quality assessment by the professionals is one of the most effective preventive actions within the context of materials management in the hospital environment, since it prevents acquisition of low-quality products.
\end{abstract}

Keywords: Quality Inspection, Shopping, Hospital, Security Management, Materials Management in hospital.

\title{
RESUMEN
}

Dirigido a identificar las razones de denegaciones de materiales médicos (MM) en el aviso con invitación a licitación 001 y 029-2011 en licitación electrónica, e identificar las categorías profesionales involucrados en la evaluación de la calidad de los materiales médicos. Es un estudio cuantitativo de carácter descriptivo, transversal y documental. Los datos se obtuvieron de los formularios utilizados por profesionales para determinar la calidad de tales materiales en el aviso con la invitación a ofertar en la subasta electrónica 001 y 029 2011, presentes en el archivo de la Comisión de normalización de materiales y productos del hospital de la Universidad del norte de Minas Gerais. Se observó que los terrenos de denegaciones de la MM relación con baja calidad técnica del producto y la enfermera profesional constituye un punto fuerte en esos dos procesos licitatorios. La estrategia de presentar el MM en fase previa adquisición para evaluación de la calidad de los profesionales es una de las acciones preventivas más eficaces dentro del contexto de la gestión de materiales en el ámbito hospitalario, ya que previene la adquisición de productos de baja calidad.

Palabras clave: Inspección de Calidad, Compras, Hospital, Gestión de la Seguridad, Gestión de Materiales en el hospital.

\section{INTRODUÇÃO}

A gestão de materiais no setor saúde, desde sua aquisição até sua utilização, consome uma parte significativa de recursos financeiros (em torno de $20 \%$ do total). Diante disso, implantar metodologias que asseguraram o controle efetivo deste processo é relevante para se evitar gastos desnecessários e zelar por uma qualidade da atenção. O gerenciamento de materiais tem como intuito alocar os recursos imprescindíveis ao processo produtivo com qualidade e quantidades adequadas, no momento certo e de forma efetiva. Entende-se por materiais médico-hospitalares $(\mathrm{MMH})$, os artigos de consumo, implantáveis, órteses, próteses, artigos descartáveis, produtos 

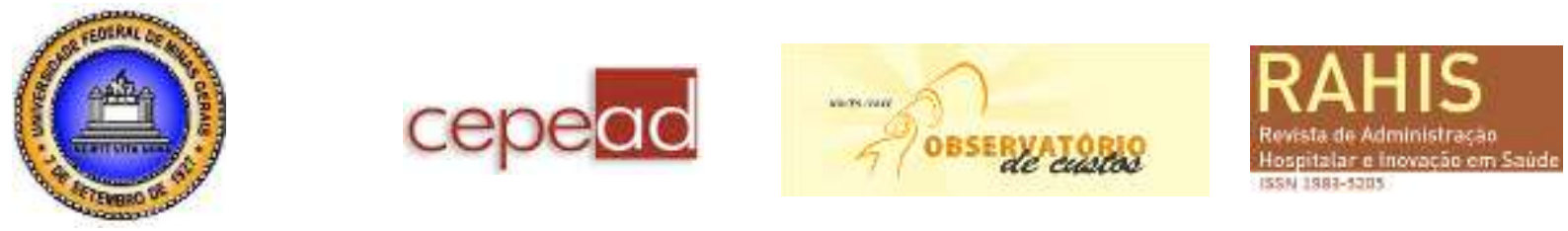

químicos, instrumental médico cirúrgico de pequeno porte, e todo aquele que por ventura for utilizado e seguir com o paciente ou for descartado após o uso (BRASIL, 2007).

Freire et al. (2012) reforçam que uma boa gestão dos equipamentos médicos pode proporcionar processos mais efetivos, tanto pela aquisição de novas tecnologias no lugar de outras mais antigas, quanto pela introdução de novas tecnologias ou equipamentos de integração. Segundo estes mesmo autores, o acesso ao estado da arte no tratamento médico aumenta as esperanças de cura na visão dos pacientes.

A introdução no Brasil, em 2001, do conceito de Gerenciamento de Risco Hospitalar pela Agência Nacional de Vigilância Sanitária (Anvisa), do Ministério da Saúde, com o projeto Hospitais Sentinela, permitiu a adoção de medidas de valorização da qualidade da atenção em saúde (KUWABARA, EVORA e OLIVEIRA, 2010).

O gerenciamento de risco, uma preocupação recente entre hospitais e profissionais de saúde, consiste em um processo complexo de ações multidisciplinares que tem como finalidade identificar e prevenir eventos adversos. A gestão de riscos garante a qualidade da assistência e segurança do paciente no que concerne a medicamentos (farmacovigilância); materiais e equipamentos médicohospitalares (tecnovigilância); saneantes; e sangue e hemoderivados (hemovigilância) (KUWABARA, EVORA e OLIVEIRA, 2010).

Dentro deste contexto, faz-se necessário associar ao processo de gerenciamento de risco, o componente humano, como prima a Qualidade Total, que é uma forma de gestão na qual as pessoas são consideradas elementos importantes para o sucesso da empresa. O principio básico da qualidade é o contentamento de todos os envolvidos na empresa: colaboradores, fornecedores e clientes. Implantar um programa de qualidade total constitui em uma forma de buscar melhorias continuas no atendimento, na diminuição de custos, na minimização de desperdícios e, implicando, na eliminação de erros (TAJRA, 2010).

A Comissão de Padronização de Materiais e Produtos do Hospital Universitário Clemente de Faria (COPAMP/HUCF) da Universidade Estadual de Montes Claros - UNIMONTES - tem a finalidade de formular e implementar uma política institucional para o uso racional e de qualidade de materiais e equipamentos médico-hospitalares, objetivando contribuir para a melhoria da assistência prestada em saúde. A comissão avalia periodicamente os materiais e equipamentos padronizados, promovendo inclusões e exclusões segundo critérios de eficácia, eficiência clínica e custo, por meio também da abordagem de usuários do serviço hospitalar para o preenchimento de um formulário confeccionado para esta finalidade. Dentre suas principais atribuições tem-se a realização das avaliações, a elaboração da relação de materiais e equipamentos médico-hospitalares padronizados e o desenvolvimento de ações de tecnovigilância.

Neste sentido, a COPAMP/HUCF juntamente com o usuário final de tais produtos vem atuando na avaliação dos MMH. Identificar os motivos de reprovações constitui em uma forma de reconhecer a necessidade demandada pelos profissionais de saúde, bem como proporcionar informações que permita aos fornecedores adequarem-se para que os atendam e que seja um processo consoante às exigências dos editais licitatórios.

Por licitação entende-se o processo formal de aquisição realizado por órgãos públicos, conforme os preceitos legais estabelecidos para tal fim, com o objetivo de atender às necessidades da organização quanto à compra de produtos, dedicado ao processo produtivo. No Brasil as licitações estão regulamentadas pela Lei 8.666 de 21 de junho de 1993, atualizada pelas leis 8.883 de 8 de junho de 1994 e 9.648 de 27 de maio de 1998 (VECINA NETO e REINHARDT FILHO, 1998). 

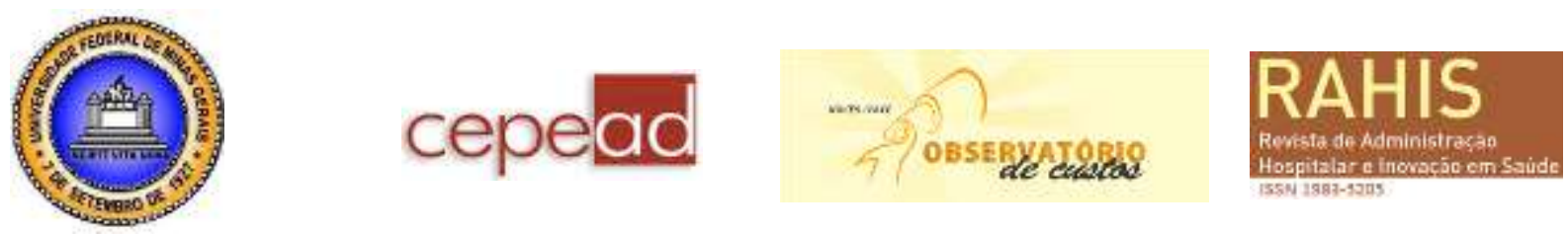

Assim, o presente estudo visou identificar os motivos de reprovações de materiais médicohospitalares no edital de licitação 001 e 029/2011, na modalidade pregão eletrônico do COPAMP/HUCF e identificar as categoriais profissionais envolvidas na avaliação da qualidade dos materiais médico-hospitalares.

\section{REFERENCIAL TEÓRICO}

O Projeto Hospital Sentinela foi criado pela Agência Nacional de Vigilância Sanitária (ANVISA), e constitui uma rede nacional de hospitais com a finalidade de notificar ativa e qualificadamente queixas técnicas e efeitos adversos relacionados a produtos de saúde: materiais médico-hospitalares, equipamentos eletroeletrônicos, medicamentos, sangue e hemoderivados, dentre outros. O Projeto segue três diretrizes: (1) Tecnovigilância - detecção de eventos adversos relacionados a equipamentos, artigos de uso médico e até saneantes e materiais de limpeza; (2) Farmacovigilância - identificação e avaliação dos efeitos adversos de medicamentos em pacientes expostos a tratamentos específicos; (3) Hemovigilância - detecção, obtenção de dados e análise dos efeitos indesejáveis de transfusão de sangue em todo o seu processo (CHAVES, 2002).

Embora seja de suma importância a implementação de programas de gerenciamento de risco a fim de garantir as condições mínimas de segurança dentro dos ambientes hospitalares, isso ainda não é uma realidade comum nos hospitais brasileiros. Dentre os principais fatores dificultadores para a sua disseminação está o desconhecimento dos profissionais acerca das técnicas e conceitos envolvidos neste processo. Assim, difundir a discussão sobre o tema se torna premente (FLORENCE e CALIL, 2005).

Com a constante incorporação de tecnologias na saúde, o gerenciamento de risco hospitalar deve ser inserido para sistematizar o monitoramento de eventos adversos, desenvolvendo ações para o controle e eliminação de seus danos. Assim, a tecnovigilância age nos procedimentos para aquisição, utilização e controle da qualidade de produtos e equipamentos na saúde (KUWABARA, EVORA e OLIVEIRA, 2010).

A criação de um comitê permanente de avaliação técnica de materiais e medicamentos composta por usuários finais ou representantes destes permite avaliar e supervisionar o padrão de qualidade estabelecido (PAULUS JÚNIOR, 2005).

Nessa direção, a qualidade é requisito fundamental, que proporciona a segurança necessária para os resultados demandados. Implantar metodologia de avaliação de um produto médicohospitalar na fase pré-aquisição é relevante e se torna premente, considerando que eventos adversos podem ocorrer mesmo com produtos considerados de boa qualidade (KUWABARA, EVORA e OLIVEIRA, 2010).

A pré-qualificação é uma forma de realizar a verificação da conformidade dos produtos possíveis de licitar com o contrato de registro sanitário para refinar a especificação do edital da licitação. Esta estratégia vem sendo utilizada por hospitais brasileiros. O processo envolve a obtenção de uma série de informações técnicas e sanitárias e a realização de testes funcionais em amostras de diversas marcas e modelos, na qual vai possibilitar ou não a decisão de compra. Tornase crucial o conhecimento aprofundado das características técnicas dos produtos e das finalidades a que se destinam para que os editais sejam elaborados (TRINDADE et al., 2010).

Uma organização de saúde é um sistema produtivo de atenção à saúde, na qual o setor de abastecimento integra-se como subsistema para atender as necessidades de insumos (materiais de consumo) e de equipamentos (materiais permanentes) dos profissionais de saúde que desenvolvem a sua assistência (INFANTE e SANTOS, 2007). 

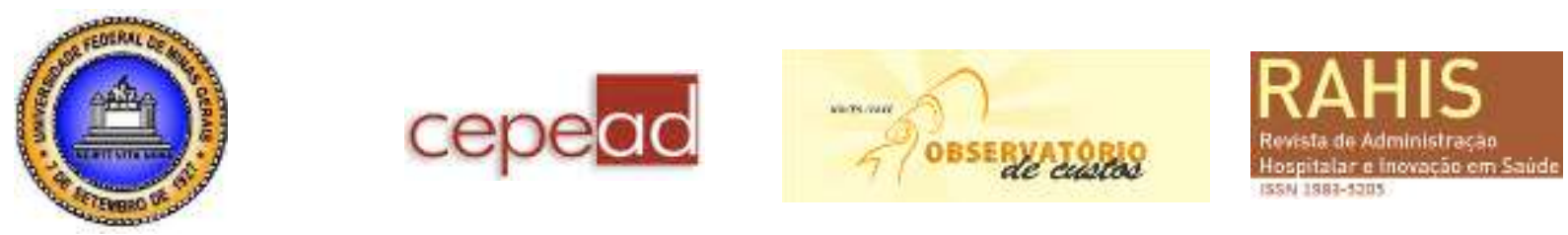

A intenção no setor público sugere diminuir os problemas de abastecimento dos serviços de saúde dada à insuficiência de recursos orçamentários. Os efeitos deletérios, como o comprometimento da assistência, consequentes dos períodos de desabastecimento não devem passar despercebido. No entanto, são notórios os desperdícios e a má utilização de insumos e equipamentos, a escassa qualificação dos profissionais da área de abastecimento e a pouca atenção ao planejamento logístico nas organizações públicas de saúde (INFANTE e SANTOS, 2007).

Os profissionais envolvidos no gerenciamento das unidades hospitalares - médicos, enfermeiros, nutricionistas, farmacêuticos, engenheiros, entre outros, devem possuir conhecimento que os possibilite tomar decisão e alocação de recursos, uma vez que estes são geradores de custos. Para isso, devem determinar quais recursos são necessários para o desenvolvimento de atividades assistenciais ou de apoio, levando em consideração não apenas os aspectos quantitativos e qualitativos, mas, também, os aspectos financeiros, visto que esses causam impacto para a organização (LOURENCO e CASTILHO, 2006).

Tanto os produtos oferecidos em organizações de saúde são complexos e exige elevada qualificação profissional, como também, os insumos utilizados são cada vez mais sofisticados e numerosos (INFANTE e SANTOS, 2007). Neste contexto, o descritivo da tecnologia e a especificação técnica assumem o papel primordial no edital de contratação da compra (TRINDADE et al., 2010).

As compras públicas exigem um formalismo maior dado à previsão legal que o administrador público deve seguir, sendo regidas pela Lei 8.666 de 21 de junho de 1993, alterada pelas Leis 8.883/94, 9.032/95 e 9.648/98. Mais recentemente a Lei 10.520/02 introduz a modalidade de compras através do pregão, uma vez que a licitação visa à proposta mais vantajosa para a administração pública (PAULUS JÚNIOR, 2005).

A modalidade de licitação pregão é uma forma para aquisição de bens e serviços comuns pela União, Estados, Distrito Federal e Municípios, conforme disposto em regulamento, qualquer que seja o valor estimado da contratação, na qual a disputa pelo fornecimento é feita por meio de propostas e lances, em sessão pública presencial ou eletrônica (BRASIL, 2010).

O excesso estocado de alguns materiais e a escassez de outros, dentro de um hospital, é talvez um dos pontos críticos que mais afligem os profissionais envolvidos com o processo gerencial. Se por um lado, a escassez implica na interrupção da assistência, levando a vivência de situações danosas e estressantes para o cliente, família e profissionais, por outro lado, grandes estoques de outros, ocasiona, além da perda de capital decorrente dos problemas que surgem devido à falta de controle de estoque, a falta deste mesmo capital para a compra dos demais materiais ausentes (BRASIL, 2010).

Gerenciar um grande número de itens em processos de produção distintos é imperativo, para que as instituições trabalhem com estoques. Em termos econômicos, esses têm o mesmo sentido de dinheiro guardado nas prateleiras, implicando em uma serie de consequências e risco como: ocuparem espaço, consumirem energias de conservação, terem seus prazos de validade vencidos, ficarem obsoletos, desaparecerem sem explicações e outros riscos (PAULUS JÚNIOR, 2005).

Pode-se falar que os objetivos de uma unidade de gerenciamento de materiais em hospitais e unidades de saúde visam a garantir: a) a continuidade da oferta dos serviços de saúde; b) baixos custos de aquisição, de realização do pedido e de manutenção dos estoques; c) alta rotatividade dos estoques; d) qualidade no atendimento; e) qualidade dos materiais; f) bom relacionamento com os fornecedores; g) controles cadastrais e conhecimento do mercado e dos fornecedores; h) obter o máximo retorno; i) centralizar controles mesmo com descentralização de atividades; j) padronizar o uso de materiais (PAULUS JÚNIOR, 2005). 

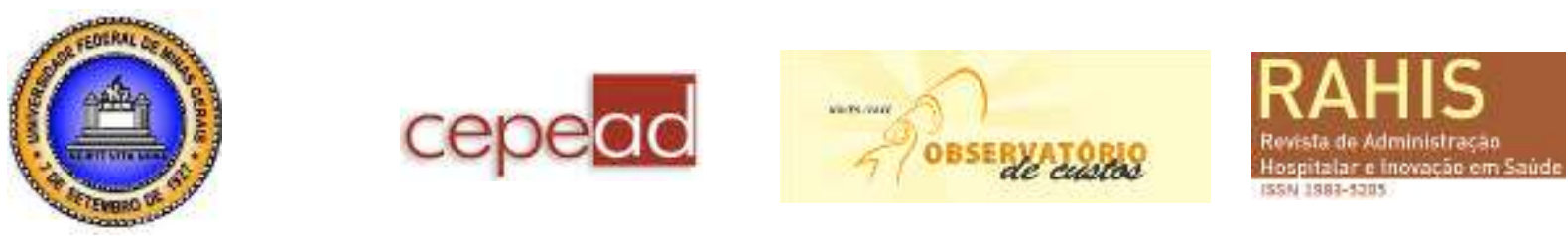

Pessoas com níveis de conhecimento e informações diferentes têm diferentes entendimentos sobre o que veem e pensam, ou seja, pode ser claro e comum para quem está usando o material e solicitando a aquisição e ser incompreensível para quem está comprando. Dessa forma, o resultado das negociações de compras envolvendo itens com especificações incompletas, pode levar a consequências como a aquisição de um objeto inútil para o setor requisitante. A Padronização dos itens de uso frequentes reduz os problemas decorrentes da especificação incompleta (PAULUS JÚNIOR, 2005). Assim, Michelin et al. (2005) afirmam que o manual de material-médicohospitalar é uma ferramenta de fundamental importância no processo de gestão da farmácia hospitalar, uma vez que fornece informações sobre os artigos hospitalares padronizados dentro da instituição de forma ordenada, e servindo de consulta por toda equipe técnica.

\section{MÉTODOS}

Trata-se de um estudo descritivo, documental, com abordagem quantitativa, realizado junto à Comissão de Padronização de Materiais e Produtos do Hospital Universitário Clemente de Faria, em Montes Claros, norte de Minas Gerais - Brasil.

Os dados foram obtidos a partir dos formulários utilizados pelos funcionários para averiguar a qualidade de tais materiais, referentes ao edital de licitação na modalidade pregão eletrônico 001 e 029/2011 da COPAMP/HUCF. Utilizaram-se todos os formulários que se encontravam arquivados no referido setor. As variáveis de interesse foram agrupadas por categorias, organizadas em um banco de dados, no programa Microsoft Office Excel 2007 e submetidas à análise descritiva, que viabilizou a confecção de quadros e gráficos.

Este trabalho foi submetido à apreciação da Diretoria Acadêmica do Hospital Universitário Clemente de Faria, obtendo parecer favorável.

\section{RESULTADOS}

No ano de 2011 foi realizado o processo licitatório na modalidade pregão eletrônico 001 que permitiu a aquisição de 458 itens distribuídos em 199 lotes. A análise dos formulários permitiu concluir que amostras de 86 lotes foram submetidas à avaliação da qualidade, representando, portanto $43 \%$.

Neste processo licitatório constatou-se que o profissional Enfermeiro esteve presente em $57,4 \%$, seguida pela categoria médica, presente em $56,4 \%$ dos testes. Também se observou a presença de outras categorias profissionais como a fisioterapia, nutrição, farmacêutico e técnico de manutenção de equipamentos médicos (Gráfico 1). 


\section{cepead}
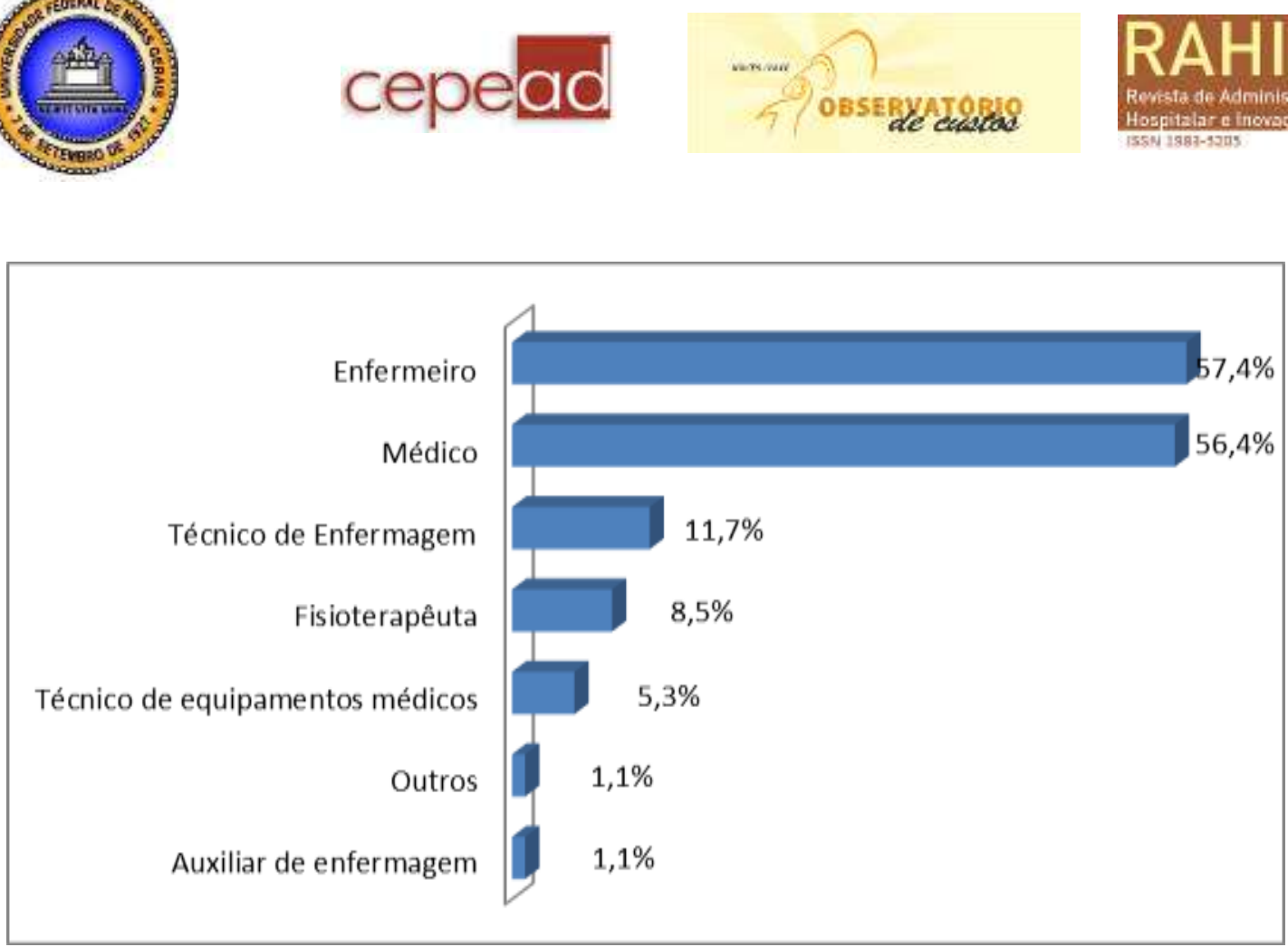

Gráfico 1 - Distribuição da categoria profissional envolvidas na avaliação da qualidade dos materiais-médico-hospitalares no pregão 001/2011.

Foi possível identificar 60 justificativas de reprovação dos $\mathrm{MMH}$, sendo 20 destas, atribuídas à amostra incompatível com as especificações do item no edital. Entre as amostras estavam atadura de crepom, curativo, compressa, fio de sutura entre outros. Assim, a não conformidade com o edital representou $33 \%$ dos motivos de reprovações.

No ano de 2011 também foi realizado o processo licitatório na modalidade pregão eletrônico 029, que permitiu a aquisição de 128 itens distribuídos em 64 lotes. A análise dos formulários permitiu inferir que amostras de 42 lotes foram submetidas à avaliação da qualidade, representando, portanto $66 \%$.

Tendo em vista a caracterização da participação das categorias profissionais na avaliação da qualidade, observou-se que no pregão eletrônico 029/2011 o profissional Enfermeiro esteve presente em 41,5\% dos testes (Gráfico 2).

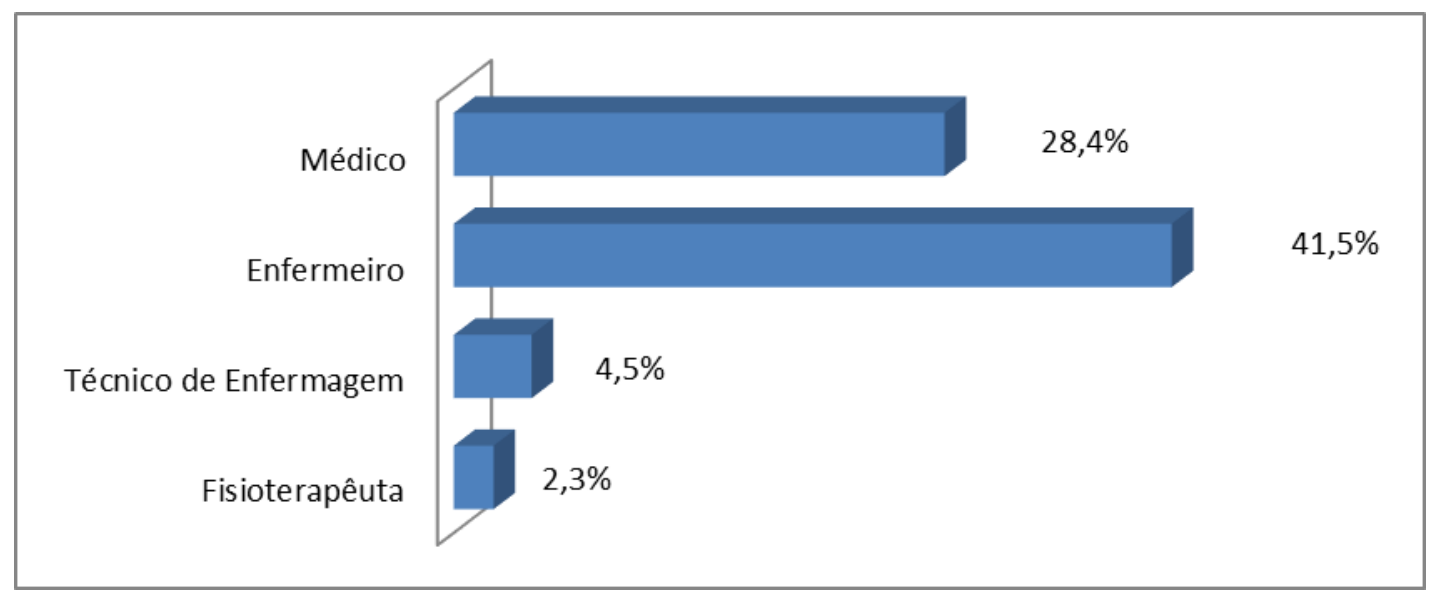

Gráfico 2 - Distribuição da categoria profissional envolvidas na avaliação da qualidade dos materiais-médico-hospitalares no pregão 029/2011. 


\section{cepead}
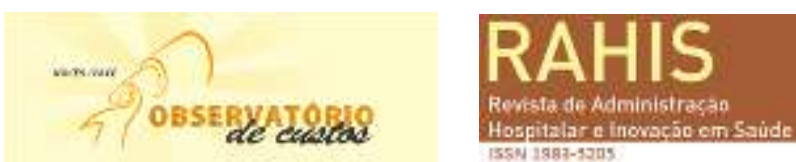

Neste processo licitatório foi possível contabilizar 34 motivos de reprovação dos $\mathrm{MMH}$, sendo 21 reprovações devidas à amostra incompatível com as especificações do item no edital, assim como ocorreu com o pregão 001/2011. Dentre as amostras estavam atadura de crepom, bolsa de colostomia, equipo para solução parenteral e fio de sutura. Dessa forma, a não conformidade com o edital representou $62 \%$ dos motivos de reprovações.

Tendo em vista que as demais justificativa de reprovação do MMH têm uma característica peculiar de acordo com o material, observou-se a necessidade de construção de um quadro que contemplasse tais motivos, nos pregões 001 e 029/11 (QUADRO 1). A análise global permite dizer que de forma sintética os motivos de reprovações estavam diretamente ligados a baixa qualidade técnica do produto.

Quadro 1 -Justificativa de reprovações dos MMH segundo o pregão eletrônico no ano de 2011.

\begin{tabular}{|c|c|c|}
\hline Material & $\begin{array}{c}\text { Justificativas pregão } \\
029 / 11\end{array}$ & Justificativas pregão 001/11 \\
\hline \multirow{2}{*}{$\begin{array}{l}\text { Agulha para } \\
\text { anestesia raque }\end{array}$} & \multirow{2}{*}{$\begin{array}{l}\text { Ao introduzir no paciente } \\
\text { há entorse desta, } \\
\text { prejudicando o } \\
\text { procedimento. }\end{array}$} & $\begin{array}{l}\text { Demora voltar liquor no bisel aumentando tempo de } \\
\text { procedimento e colocando sempre em dúvida a eficácia }\end{array}$ \\
\hline & & Baixa qualidade técnica, com risco de falhas aumentadas. \\
\hline Agulha descartável & 1 & $\begin{array}{l}\text { Agulha solta facilmente do bico da seringa. Isso pode } \\
\text { levar o profissional acidentar. Dificuldade em retirar a } \\
\text { agulha da capa protetora }\end{array}$ \\
\hline $\begin{array}{l}\text { Cânula de } \\
\text { traqueostomia. }\end{array}$ & Fixação ruim. & e \\
\hline $\begin{array}{l}\text { Cateter venoso } \\
\text { central de inserção } \\
\text { periférica }\end{array}$ & $\begin{array}{l}\text { Introdutor é muito } \\
\text { calibroso e rígido }\end{array}$ & $\begin{array}{l}\text { Cateter extremamente rígido de difícil progressão } \\
\text { intravenoso e com elevada taxa de infiltração }\end{array}$ \\
\hline $\begin{array}{l}\text { Cateter intravenoso } \\
\text { central }\end{array}$ & e & $\begin{array}{l}\text { Difícil progresso do cateter. Fixação externa difícil, com } \\
\text { permanência da agulha, inclusive, com risco de } \\
\text { perfuração do cateter. Agulha exagerada com trauma } \\
\text { vascular. Menor custo benefício pelo uso frequente de } \\
\text { vários cateteres para o mesmo paciente }\end{array}$ \\
\hline Fita termo sensível & $\begin{array}{l}\text { Não possui boa fixação, } \\
\text { não possui uniformidade } \\
\text { na mudança de cor. Baixa } \\
\text { sensibilidade a autoclave. }\end{array}$ & $\begin{array}{l}\text { Aderência ruim, soltando com facilidade após sair da } \\
\text { autoclave. Baixa sensibilidade ao calor úmido. }\end{array}$ \\
\hline Seringa para insulina & $\begin{array}{l}\text { A agulha dobra-se com } \\
\text { facilidade }\end{array}$ & - \\
\hline $\begin{array}{l}\text { Torneira cirúrgica } \\
\text { descartável. }\end{array}$ & $\begin{array}{l}\text { Difícil manuseio, facilita } \\
\text { a contaminação do } \\
\text { sistema; }\end{array}$ & - \\
\hline $\begin{array}{l}\text { Tubo endotraqueal: } \\
\text { balonetes }\end{array}$ & $\begin{array}{l}\text { Baixa resistência dos } \\
\text { balonetes e conexões }\end{array}$ & - \\
\hline $\begin{array}{l}\text { Eletrodo para } \\
\text { eletrocardiograma }\end{array}$ & Não captação de ondas & $\begin{array}{l}\text { O produto testado não apresentou aderência necessária } \\
\text { para monitorização do paciente }\end{array}$ \\
\hline Luva cirúrgica & - & $\begin{array}{l}\text { Pouca elasticidade e problemas no empacotamento, } \\
\text { dificultando o uso adequado. }\end{array}$ \\
\hline
\end{tabular}



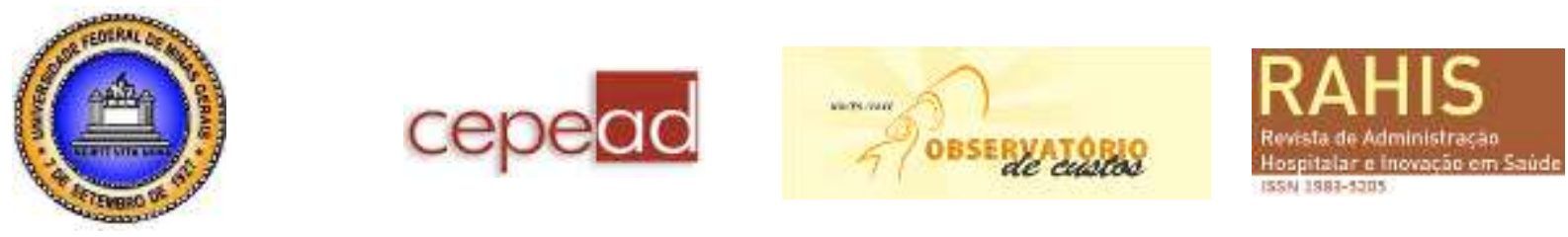

\begin{tabular}{|l|c|l|}
\hline $\begin{array}{l}\text { Manguito de pressão } \\
\text { não invasiva }\end{array}$ & - & $\begin{array}{l}\text { Revestimento interno impede a desinfecção adequada } \\
\text { Não foram enviadas instruções de uso em português. }\end{array}$ \\
\hline Fio de sutura & - & Fio soltou facilmente da agulha \\
\hline
\end{tabular}

Legenda: "“_": Item não adquirido no Pregão.

\section{DISCUSSÃO}

Garcia et al (2012) afirmam que para que se ofereça uma assistência de qualidade é necessário que haja integração entre as ações gerenciais e assistenciais, em que o enfermeiro necessita atualizar-se quanto aos aspectos fundamentais na unidade, como a escolha do material médico-hospitalar. A classe profissional de enfermagem é um dos maiores usuários dos artigos médico-hospitalares justificando, assim, o enfermeiro envolver-se ativamente com a administração dos recursos materiais (ROGANTE e PADOVEZE, 2005).

É importante que o profissional ratifique características de dimensões apresentadas pelo fabricante nas amostras recebidas para avaliação da qualidade e testes funcionais (peso, comprimento, entre outros), visto que nem sempre o produto fornecido é coerente com às especificações que o fabricante informa na embalagem do produto (ROGANTE e PADOVEZE, 2005).

A especificação de um produto é a caracterização descritiva utilizando critérios que possibilite o reconhecimento tanto por parte dos clientes internos quanto externos. O cuidado dedicado à elaboração de uma especificação pode evitar surpresas que somente serão percebidas no momento em que o cliente se deparar frente a frente com seu material (GUIMARÃES, 2005). O departamento de compras no setor saúde e de pesquisa deve agregar a tarefa de busca constante de melhoria quanto à especificação de materiais, resultando não apenas um produto econômico e de qualidade para o setor solicitante, mas também pressupondo um mercado interessante para o fornecedor vender ou disponibilizar determinado serviço (CARVALHO, 2009).

Considerando a complexidade das organizações hospitalares, com procedimentos diversificados, e a incorporação de novas tecnologias, o controle desses insumos e seus custos são essenciais (HONÓRIO e ALBUQUERQUE, 2005). Ao introduzir novos materiais na assistência é necessário conhecê-los, avaliá-los e selecioná-los juntamente com usuários de tais produtos. Para que isso ocorra, o enfermeiro de recursos materiais é responsável pelas análises desses novos produtos, e deve ter uma rotina de atendimento aos fornecedores de produtos médico-hospitalares, para fazer triagem de qualidade e seleção de tais produtos a fim de posteriormente realizar as avaliações. O quantitativo de itens consumido frequentemente em uma unidade hospitalar representa de 3.000 a 6.000 itens. Esses números expõem a complexidade do processo produtivo (ROGANTE e PADOVEZE, 2005).

Envolvendo-se com a equipe multiprofissional o enfermeiro descentraliza o poder de decisão na aquisição e avaliação do artigo médico-hospitalar, contribuindo, assim, para a melhoria na administração dos recursos materiais (ROGANTE e PADOVEZE, 2005). Considerando a presença impactante da enfermagem evidenciada pelos dados esse mesmo autor aponta que a enfermagem precisa aperfeiçoar sua competência no processo de avaliação, seleção e aquisição de materiais, para atuar de maneira ativa na otimização dos resultados, maximização dos recursos e com considerável melhoria na qualidade da assistência prestada.

É correto que a qualidade desses instrumentos influencia a qualidade da assistência prestada e com profundas repercussões sobre a segurança do trabalhador de enfermagem ao realizar o 

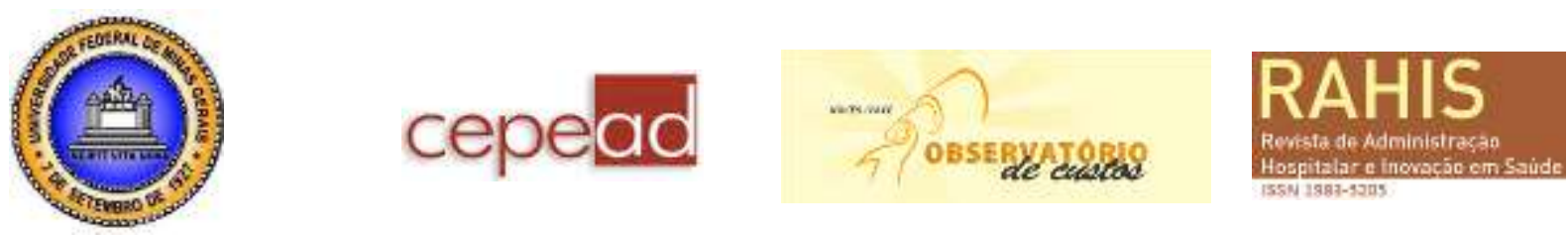

cuidado (HONÓRIO e ALBUQUERQUE, 2005). O enfermeiro deve racionalizar os recursos, visando alcançar resultados coerentes esperando oferecer a melhor assistência ao paciente ao utilizar os recursos que a instituição dispõe. É imprescindível respeitar a segurança do paciente e garantir um padrão de qualidade dos cuidados prestados (ROGANTE e PADOVEZE, 2005).

A Gestão de Recursos Materiais (GRM) nos hospitais é um ponto importante, visto que envolve não só uma diversidade desses materiais como que também esta diretamente ligada à assistência dos pacientes. Geralmente, a administração de tais materiais é feita pela área administrativa sem a inclusão das áreas técnicas (HONÓRIO e ALBUQUERQUE, 2005). O profissional enfermeiro administrador de recursos materiais que além de sua qualificação (conhecimento teórico e prático), tem a tarefa de selecionar os materiais hospitalares observando o seu custo e beneficio, procurando a otimização dos recursos financeiros, com a intenção de adquirir os melhores produtos e equipamentos destinados aos pacientes (ROGANTE e PADOVEZE, 2005). Daí a necessidade de comunicação entre os profissionais que dispõe do conhecimento técnico dessas tecnologias e o setor que atua diretamente na aquisição dos materiais.

\section{CONCLUSÃO}

Os dados deste estudo mostraram que no pregão 001 houve aquisição de 458 itens distribuídos em 199 lotes e que 43\% da amostra foram submetidas à avaliação da qualidade, e no pregão 029, houve aquisição de 128 itens e que 66\% da amostra foram submetidas à avaliação da qualidade. Em relação à categoria profissional envolvida no processo licitatório, o enfermeiro foi o profissional que mais esteve presente. Quanto aos motivos de reprovações, em termos gerais, conclui-se que tais reprovações estavam diretamente ligados a baixa qualidade técnica do produto.

O estudo em questão aponta esta estratégia de submeter os MMH em fase de pré-aquisição à avaliação da qualidade junto aos profissionais, uma das ações mais preventivas e eficazes dentro do contexto da administração de materiais no ambiente hospitalar, uma vez que previne aquisição de produtos de baixa qualidade, evitando o desperdício de recurso no setor saúde, bem como riscos aos profissionais e clientes. Tal avaliação constitui-se ainda em uma barreira formal aos itens que estão incoerentes com a especificação do edital de licitação devendo, portanto, ser reforçada.

\section{REFERÊNCIAS}

BRASIL. Agência Nacional de Vigilância Sanitária. Unidade de Tecnovigilância. Núcleo de Gestão do Sistema Nacional de Notificação e Investigação em Vigilância Sanitária. Pré-qualificação de artigos médico-hospitalares: estratégia de vigilância sanitária de prevenção. Brasília: Agência Nacional de Vigilância Sanitária, 2010.

BRASIL. Governança no Sistema Único de Saúde (SUS) do Brasil: Melhorando a Qualidade do Gasto Público e Gestão de Recursos. Relatório No. 36601-BR. 15 de fevereiro de 2007. Disponível em: $\quad \quad$ http://siteresources.worldbank.org/BRAZILINPOREXTN/Resources/38171661185895645304/4044168-1186326902607/19GovernancaSUSport.pdf $>$. Acesso em 11 de junho de 2012.

CARVALHO, A.L. Compras governamentais: uma proposta de dinamização do fluxo de compras em instituições públicas em saúde. [Dissertação]. Escola Nacional de Saúde Pública Sérgio Arouca. Fundação Oswaldo Cruz. Rio de Janeiro, 2009. 

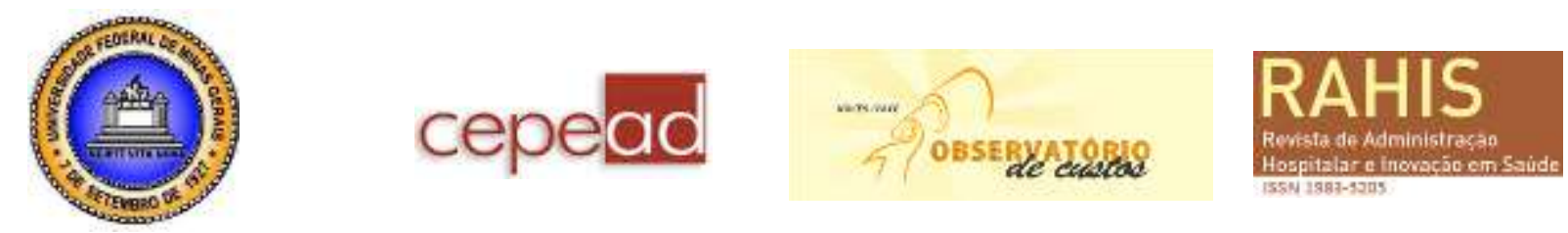

CHAVES, S. Projeto Hospital Sentinela. Revista Médica, v.36, n.2/3, 01 tela, 2002. Disponível em: <http://www.hse.rj.saude.gov.br/profissional/revista/36b/vigilancia.asp>. Acesso em 04 de abril de 2013.

FREIRE, R.P. et al. Gestão de equipamentos médicos: o papel das práticas de qualidade em um hospital de excelência brasileiro. Revista de Administração Hospitalar e Inovação em Saúde, v.8, n.8 p.30-43. Disponível em: http://web.face.ufmg.br/face/revista/index.php/rahis/article/view/1662/985>. Acesso em 01 de abril de 2013.

FLORENCE, G.; CALIL, S.J. Uma nova perspectiva no controle dos riscos da utilização de tecnologia médico-hospitalar. Rev Multi Ciência, v.5, 14 telas, 2005. Disponível em: <http://www.multiciencia.unicamp.br/artigos_05/a_04_05.pdf $>$. Acesso em 15 de dezembro de 2012.

GARCIA, S.D. et al. Gestão de material médico-hospitalar e o processo de trabalho em um hospital público. Rev. bras. enferm., v.65, n.2, p.339-46, 2012.

GUIMARÃES, A.L.F. Gestão e racionalização na distribuição de medicamentos e materiais clínicos: um estudo de caso no Hospital Escola da Universidade de Taubaté. [Dissertação]. Departamento de Economia, Contabilidade e Administração, Universidade de Taubaté. Taubaté, 2005.

HONÓRIO, M.T; ALBUQUERQUE, G.L. A gestão de materiais em enfermagem. Ciência, Cuidado e Saúde, v.4, n.3, p.259-68, 2005

INFANTE, M.; SANTOS, M.A.B.A. Organização do abastecimento do hospital público a partir da cadeia produtiva: uma abordagem logística para a área de saúde. Ciênc. saúde coletiva, v.12, n.4, p.945-54, 2007.

KUWABARA, C.C.T.; EVORA, Y.D.M.; OLIVEIRA, M.M.B. Gerenciamento de risco em tecnovigilância: construção e validação de instrumento de avaliação de produto médico-hospitalar. Rev. Latino-Am. Enfermagem, v.18, n.5, p. 943-51, 2010.

LOURENCO, K.G.; CASTILHO. V. Classificação ABC dos materiais: uma ferramenta gerencial de custos em enfermagem. Rev. bras. enferm., v.59, n.1, p.52-5, 2006.

MICHELIN, A.F. et al. Gestão de material medico hospitalar em um serviço de farmácia de um hospital geral e elaboração de um manual de material medico hospitalar. Infarma, v.17, n.3/4, p.668, 2005. Disponível em: 〈http://www.cff.org.br/sistemas/geral/revista/pdf/17/gestao_material.pdf〉. Acesso em 21 de fevereiro de 2013.

PAULUS JÚNIOR, A. Gerenciamento de Recursos Materiais em Unidades de Saúde. Revista Espaço para a Saúde, v.7, n.1, p.30-45, 2005.Disponível em: <http://www.ccs.uel.br/espacoparasaude/v7n1/Gerenciamento.pdf $>$. Acesso em 14 de dezembro de 2012. 
ROGANTE, M.M.; PADOVEZE, M.A. Padronização, qualificação e aquisição de materiais $e$ equipamentos médico-hospitalares. São Paulo: EPU, 2005.

TAJRA, S.F. Gestão estratégica na saúde: reflexões e praticas para uma administração voltada para excelência. São Paulo: Iatria, 2010.

TRINDADE, E. et al. Estudo multicêntrico de pré-qualificação: estudo de caso sobre equipos. BIT - Boletim Informativo de Tecnovigilância, n. esp., p.1-14, 2010. Disponível em: 〈http://www.anvisa.gov.br/boletim_tecno/boletim_tecno_dez_2010/PDF/Ed1_especial/todos.pdf $>$. Acesso em 14 de dezembro de 2012.

VECINA NETO, G.; REINHARDT FILHO, W. Gestão de recursos materiais e de medicamentos. São Paulo: Faculdade de Saúde Pública da USP; 1998. 